\title{
Comparison of Safety and Efficacy of Intrastromal Injections of Voriconazole, Amphotericin B and Natamycin in Cases of Recalcitrant Fungal Keratitis: A Randomized Controlled Trial
}

\author{
Gunjan Saluja' \\ Namrata Sharma (D) \\ Rinky Agarwal' \\ H P Sharma ${ }^{2}$ \\ Deepali Singhal' \\ Prafulla Kumar Maharana' \\ Rajesh Sinha' \\ Tushar Agarwal' \\ T Velpandian ${ }^{2}$ \\ Jeewan S Titiyal' \\ Gita Satpathy ${ }^{3}$ \\ 'Department of Ophthalmology, \\ Dr. Rajendra Prasad Centre for \\ Ophthalmic Sciences, All India Institute of \\ Medical Sciences, New Delhi, India; \\ ${ }^{2}$ Ocular Pharmacology and Pharmacy \\ Division, Dr. Rajendra Prasad Centre for \\ Ophthalmic Sciences, All India Institute of \\ Medical Sciences, New Delhi, India; \\ ${ }^{3}$ Department of Ocular Microbiology, \\ Dr. Rajendra Prasad Centre for \\ Ophthalmic Sciences, All India Institute of \\ Medical Sciences, New Delhi, India
}

\begin{abstract}
Objective: To compare the safety and efficacy of intrastromal voriconazole (IS-VCZ), amphotericin B (IS-AMB) and natamycin (IS-NTM) as an adjunct to topical natamycin (NTM) in cases of recalcitrant fungal keratitis.

Design: Prospective randomized trial.

Setting: Tertiary eye centre.

Participants: Sixty eyes of 60 patients with microbiologically proven recalcitrant fungal keratitis (ulcer size $>2 \mathrm{~mm}$, depth $>50 \%$ of stroma, and not responding to topical NTM therapy for two weeks) were recruited.

Methods: patients were randomized into three groups of 20 eyes, each receiving ISVCZ 50ug/ $0.1 \mathrm{~mL}$, ISAMB, $5 \mathrm{ug} / 0.1 \mathrm{~mL}$ and ISNTM $10 \mathrm{ug} / 0.1 \mathrm{~mL}$ (prepared aseptically in ocular pharmacology). The patients in all three groups continued topical NTM 5\% every four hours until the ulcer healed. Primary outcome measure was time taken till complete clinical resolution of infection, and secondary outcome measure was best corrected visual acuity (BCVA) at six months.

Results: All three groups had comparable baseline parameters. The mean duration of healing was significantly better $(\mathrm{p}=0.02)$ in the ISNTM group ( $34 \pm 5.2$ days) as compared to the ISVCZ group ( $36.1 \pm 4.8$ days) and the ISAMB group (39.2 \pm 7.2 days). About $95 \%$, $90 \%$ and $95 \%$ patients healed successfully in the ISVCZ, ISAMB and ISNTM groups, respectively. In terms of healing, deep vascularization was significantly greater in the ISAMB group $(55 \%, \mathrm{p}=0.02)$ when compared to the ISVCZ and ISNTM groups $(31 \%$ and $26 \%$, respectively). There were fewer repeat injections in the ISNTM group ( $7 / 20 \mathrm{vs} 8 / 20$ and 9/20 in the ISVCZ and ISNTM groups, respectively).
\end{abstract}

Conclusion: Intrastromal injections are a safe and effective adjunct to conventional therapy in the management of recalcitrant fungal keratitis. ISNTM had a similar visual outcome with faster healing while ISAMB had a higher rate of deep vascularization after healing.

Keywords: intrastromal injections, recalcitrant fungal keratitis

\section{Plain Language Summary}

Department of Ophthalmology, Cornea, Cataract and Refractive Surgery Services, 4th Floor Dr. Rajendra Prasad Centre for Ophthalmic Sciences, All India Institute of Medical Sciences, Ansari Nagar East, New Delhi,

Tel $+9|-||-26593| 44$

Fax +91-II-26588919

Email namrata.sharma@gmail.com
In this randomized trial involving 60 patients with fungal keratitis, $95 \%, 90 \%$ and $95 \%$ of patients healed successfully in the ISVCZ, ISAMB and ISNTM groups; that is, there were no significant differences.

The three intrastromal injections are comparable in efficacy and treating ophthalmologists can choose from any of these as an adjunct to conventional therapy for recalcitrant fungal keratitis. 


\section{Introduction}

Fungal keratitis often presents with deep stromal abscess and endothelial plaque that needs to be managed timely and appropriately to prevent resultant ocular morbidity. ${ }^{1}$ Standard topical therapy with commonly used antifungal agents, namely, Natamycin (NTM) 5\% and Voriconazole (VCZ) 1\%, may not be sufficient for treating nonresponding fungal keratitis due to limited efficacy and the low capability of the medications to penetrate the deep layers of the cornea. Various other interventions, such as penetrating keratoplasty (PKP), collagen crosslinking with photoactivated riboflavin (PACK-CXL), excisional keratectomy combined with focal cryotherapy and amniotic membrane inlay, have been proposed to deal with these cases. ${ }^{2-4}$ However, these have their own limitations and targeted delivery of antifungal agents at the ulcer site by means of intrastromal injections remains an effective alternative of bypassing these complex options. ${ }^{5,6}$ These injections are known to enhance the drug levels at the desired site of the cornea thereby allowing successful healing of the corneal ulcer. ${ }^{5,10-16}$

Voriconazole is the most commonly used antifungal agent for intrastromal delivery (ISVCZ) and numerous studies have previously proven its efficacy in recalcitrant cases. ${ }^{6}$ Similarly, intrastromal Amphotericin-B (ISAMB) has also been employed successfully for targeted drug delivery. ${ }^{7}$ In an experimental study in rabbit eyes by Mimouni et al, intrastromal natamycin (ISNTM) was employed successfully for treatment of Fusarium keratitis. ${ }^{8}$ While many studies have individually described the utility of these drugs for intrastromal injections in recalcitrant fungal keratitis, there is a relative paucity of peer-reviewed literature prospectively comparing the safety and efficacy of these drugs.

We conducted a randomized clinical trial to determine and compare the safety and efficacy of ISVCZ 50ug/ $0.1 \mathrm{~mL}$, ISAMB $5 \mathrm{ug} / 0.1 \mathrm{~mL}$ and ISNTM $10 \mathrm{ug} / 0.1 \mathrm{~mL}$ as adjuncts to topical NTM $5 \%$ in cases of recalcitrant fungal keratitis.

\section{Methods}

The study was a prospective interventional study. The research was conducted adhering to the tenets of the Declaration of Helsinki and institutional ethics committee approval from the ethics committee of the All India Institute of Medical Sciences, New Delhi, India (IECPG106/30.12.2015) was obtained before starting the study.
The research has also been registered under the Clinical Trials Registry, India (CTRI/2021/01/030360), National Institute of Medical Statistics, ICMR, New Delhi (www. ctri.nic.in). Written informed consent was obtained from all participants.

\section{Patient Selection}

All consecutive patients presenting to our centre with unilateral fungal keratitis between January 2016 and July 2017 were screened for the following criteria: age $>18$ years, ulcer size $>2 \mathrm{~mm}$ in any dimension, involving more than $50 \%$ stromal thickness and smear or cultureproven recalcitrant fungal keratitis (Figure 1). Recalcitrant keratitis was defined as the ulcer showing no change/ increase in the size of the epithelial defect, decrease of less than $20 \%$ of stromal infiltrate or increase in hypopyon on standard topical NTM 5\% therapy for at least two weeks. The exclusion criteria were cases of mixed infection on smear or culture analysis, evidence of herpetic keratitis, impending perforation, bilateral ulcers, those with vision of less than $6 / 60$ in the fellow eye, pregnancy or breastfeeding and history of known drug allergies. Patients not compliant with medications or not ready for follow-up were excluded from the study.

After obtaining a thorough history regarding onset, progression and duration of the ulcer, a detailed history of prior ocular trauma/surgery, steroid use and drug allergies was sought. The patients were also asked about systemic comorbidities such as diabetes mellitus and drug allergies, if any. Best corrected visual acuity (BCVA) was recorded using both Snellen chart and the logarithm of the minimum angle of resolution (logMAR) scale. The values of low vision like $<1 / 60$ (logMAR 1.77$)$ were recorded as follows: counting fingers close to face 1.9 , hand movements close to face -2.3 , light perception -2.7 and no light perception $-3 .^{9}$ A detailed slit-lamp examination was undertaken by the treating ophthalmologist to note the size of epithelial defect and the stromal infiltrate. Both the parameters were measured in the greatest dimension and an axis perpendicular to it. They were expressed as mean of the longest dimension and the longest perpendicular dimension. The depth of the ulcer and the presence and height of hypopyon were also noted. Baseline clinical photography with and without fluorescein staining was performed. The posterior segment was evaluated clinically whenever possible, and B-scan ultrasonography was undertaken in all patients to rule out coexisting endophthalmitis. Following this, 


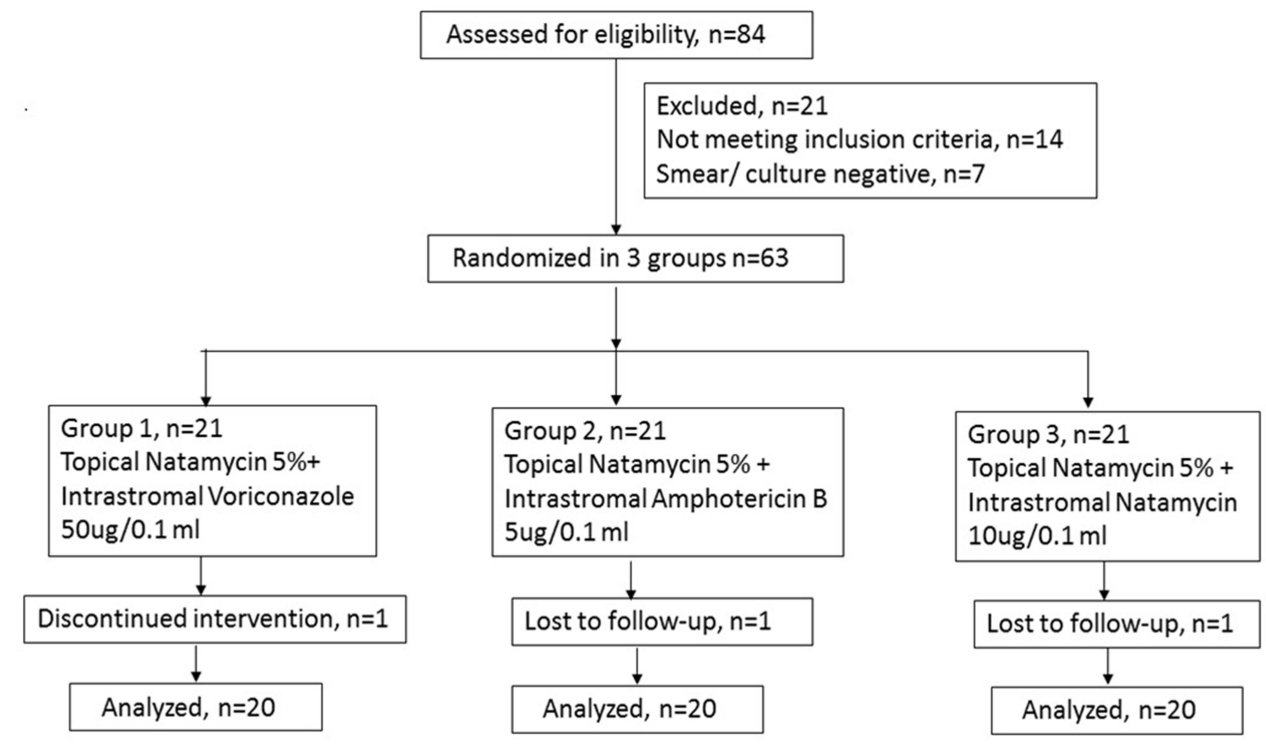

Figure I Participant flow diagram.

all patients were subjected to corneal scraping after a wash out period of 24 hours and the sample was sent for microbiological evaluation (gram staining, potassium hydroxide wet-mount preparation and culture and sensitivity on blood agar, chocolate agar, and Sabouraud dextrose agar).

Once the diagnosis of fungal keratitis was confirmed, patients were started on topical NTM 5\% two hourly and homatropine $2 \%$ four times a day. The patients were followed up for two weeks and, if the keratitis became recalcitrant, intrastromal injections were advised by the treating ophthalmologist.

Sixty eyes of 60 patients fulfilling our inclusion criteria were randomized into three groups of 20 patients each by a co-investigator using a random number table. ISVCZ, ISAMB and ISNTM were administered to group 1, 2 and 3 patients, respectively. Double-masking was performed and both the patients and the treating ophthalmologists were masked. Repeat intrastromal injections were administered after 72 hours if required.

\section{Drug Preparation}

ISVCZ $50 \mu \mathrm{g} / 0.1 \mathrm{~mL}$ was prepared by reconstituting voriconazole $200 \mathrm{mg}$ powder (Sun Pharma, Mumbai, India) with $19 \mathrm{~mL}$ Ringer's lactate. One milliliter of this solution was diluted further with $20 \mathrm{~mL}$ Ringer's lactate to obtain $0.5 \mathrm{mg} /$ $\mathrm{mL}(50 \mathrm{ug} / 0.1 \mathrm{~mL})$ solution for the intrastromal injection.

ISAMB $5 \mathrm{ug} / 0.1 \mathrm{~mL}$ was prepared by reconstituting $50 \mathrm{mg}$ of $\mathrm{AMB}$ vial with $10 \mathrm{~mL}$ of $5 \%$ dextrose. Of this solution, $0.1 \mathrm{~mL}$ was further diluted with $9.9 \mathrm{~mL}$ of $5 \%$ dextrose to achieve a concentration of $500 \mu \mathrm{g}$ in $10 \mathrm{~mL}$ $(50 \mu \mathrm{g} / \mathrm{mL}) ; 0.1 \mathrm{~mL}$ of this solution contained $5 \mu \mathrm{g}$ AMB and was employed for intrastromal injection.

ISNTM was prepared in the Department of Ocular Pharmacology, using a cyclodextrin-drug complexation technique (patent filed, available for use on request by treating clinician). The supernatant produced after overnight stirring of drug and cyclodextrin mixed in water was subjected to lyophilization. The drug content of the lyophilized powder was analyzed using the high-performance liquid chromatography method. For preparing ISNTM injection, the required amount of lyophilized powder was mixed in water, and sodium chloride was added to maintain the osmolarity of the formulation. The formulation was sterilized by passing it through a $0.22 \mu \mathrm{m}$ filter, and was dispensed in ampules. All formulation preparation processes were conducted under a laminar hood in strict aseptic conditions. The sterility of the final formulation was assured by subjecting it to bacteriological and fungal culture tests. The final formulation, found to be sterile with an osmolarity of $290 \mathrm{mOsm}$ (per liter or $\mathrm{kg}$ ), was used.

\section{Surgical Technique}

The drug was injected by the co-investigator, an experienced cornea specialist, under preferably topical anesthesia. However, for uncooperative patients peribulbar anesthesia was administered. The procedure was performed under strict aseptic precautions. After cleaning the periocular area with $10 \%$ povidone iodine and 
conjunctival sac area with $5 \%$ povidone iodine, a sterile drape was placed on the patient. The reconstituted solution was loaded in a $1 \mathrm{~mL}$ tuberculin syringe with a 30 -gauge needle. Under full aseptic conditions, the needle, with its bevel up, was inserted obliquely from the uninvolved site to reach the infiltrate at the mid-stromal level. The drug was injected at the boundaries of the infiltrate in a circumferential manner to barrage the lesion. The amount of hydration of the cornea was used as a guide to assess the area covered. On achieving the desired amount of hydration, the plunger was withdrawn slightly to ensure discontinuation of the capillary column, thus preventing back-leakage of the drug. Anterior chamber wash for hypopyon was not performed in any case.

\section{Follow-Up}

All patients were followed-up on days 1, 3, 7, 14 and 21, and every week until complete healing of the ulcer was observed or according to the discretion of the treating ophthalmologist. At each follow-up, the size and depth of the infiltrate and the ulcer size of the corneal epithelial defect and the hypopyon were measured in all patients to assess clinical response. In cases responsive to treatment, the size of the scar was also measured in its greatest dimension and presence of vascularization, if any, was noted. Intraocular pressure was also assessed digitally in all patients in the follow-up.

The primary outcome measure was time taken till complete clinical resolution of infection and the secondary outcome measure was best corrected visual acuity (BCVA) at six months. A healed case was defined as a case with complete clinical resolution of the epithelial defect, stromal infiltrate and hypopyon. Those patients who developed corneal perforation or did not show any sign of improvement after three intrastromal injections were considered as treatment failures and were started on oral antifungals (oral voriconazole, $200 \mathrm{mg}$ twice a day) and were immediately subjected to PKP based on the availability of donor corneal tissue.

These treatment failures were excluded from the analysis of final visual acuity and scar (per-protocol analysis). The last observation carry forward (LOCF) method was used to analyze the visual outcome using intention to treat analysis (ITT).

\section{Statistical Analysis}

Randomization was carried out using a computergenerated random number table. Data were recorded on a predesigned sheet and managed on an Excel spreadsheet (Microsoft, Redmond, WA). The analysis was performed using the Stata 12.1 program. Normal quantitative data analysis was conducted using ANOVA and post hoc analysis was done using the Bonferroni test. For categorical data, Fisher's exact test was used. Time to reepithelialization and resolution of infiltrates was analyzed using the Kaplan-Meier curve followed by the Cox proportional hazards model, and the results were presented as the median time to healing and hazard ratio. A p-value of $<0.05$ was deemed statistically significant.

\section{Results}

A total of 60 patients were included in the study. All three groups were age and sex-matched and demonstrated comparable baseline characteristics (Table 1). The most commonly isolated causative organism was Aspergillus sp. (32/ 60, 53.33\%), followed by Fusarium sp. (24/60, 40\%), Alternaria sp. (2/60, 3.33\%), Candida (1/60, 1.66\%) and Curvularia sp. $(1 / 60,1.66 \%)$. There were no significant differences between the three groups $(p=0.23)$ (Table 2).

\section{Primary Outcome}

In the ISVCZ, ISAMB and ISNTM groups, 19/20 (95\%), $18 / 20(90 \%)$ and19/20 (95\%) patients showed complete resolution of infection, respectively $(\mathrm{p}=0.8)$ (Table 3$)$. Of these, $12 / 20(60 \%), 11 / 20(55 \%)$ and 13/20 (65\%) patients healed with first injection; 6/20 (30\%), 5/20 $(25 \%)$ and $5 / 20(25 \%)$ healed with second injection and $1 / 20(5 \%), 2 / 20(10 \%)$ and $1 / 20(5 \%)$ healed with third injection in the ISVCZ, ISAMB and ISNTM groups, respectively. This implies that the maximum number of failures and patients needing repeat injections was seen in the ISAMB group $(p=0.8)$. Four patients $(6.66 \%)$ underwent PKP. Fifty percent of patients $(1 / 2)$ belonging to the ISAMB group and subjected to PKP developed recurrence of graft infection with the same preoperative organism and required a second PKP for complete resolution of infection. Intrastromal injections were administered at the residual host rim while performing penetrating keratoplasty; patients were also advised on topical and systemic antifungals. The corneal button was sent for histo-pathological examination, which revealed the presence of the causative fungal agent.

The average time taken for re-epithelization and for disappearance of hypopyon was significantly higher in the ISAMB group compared to the ISVCZ and ISNTM groups (Figure 2). The Kaplan-Meier curve revealed 
Table I Baseline Demographic, Microbiological and Clinical Characteristics of All Three Groups

\begin{tabular}{|c|c|c|c|c|c|}
\hline Drug & $\begin{array}{l}\text { Age (Years) } \\
\text { Mean } \pm \text { SD }\end{array}$ & $\begin{array}{l}\text { Gender } \\
\text { Distribution }\end{array}$ & $\begin{array}{l}\text { History of } \\
\text { Trauma }\end{array}$ & $\begin{array}{l}\text { Duration of Signs and Symptoms } \\
\text { (Mean } \pm \text { SD) }\end{array}$ & $\begin{array}{l}\text { Organism Traced Fu/As/ } \\
\mathrm{Ca} / \mathrm{Al} / \mathrm{Cu}\end{array}$ \\
\hline ISVCZ $(n=20)$ & $43.82 \pm 12.3$ & $12 \mathrm{M}, 8 \mathrm{~F}$ & 12 & $18.7 \pm 6.4$ days & $8 / 10 / 0 / 1 / 1$ \\
\hline ISAMB $(n=20)$ & $39.45 \pm 10.0$ & $\mathrm{I} 4 \mathrm{M}, 6 \mathrm{~F}$ & 13 & $20 \pm 5.5$ days & $8 / 10 / 1 / 1 / 0$ \\
\hline ISNTM $(n=20)$ & $40.42 \pm 10.9$ & $14 \mathrm{M}, 6 \mathrm{~F}$ & 13 & $20.8 \pm 5.1$ days & $8 / 12 / 0 / 0 / 0$ \\
\hline$p$-value & 0.41 & 0.82 & 0.8 & 0.51 & 0.23 \\
\hline
\end{tabular}

Abbreviations: ISVCZ, intrastromal voriconazole; ISAMB, intrastromal amphotericin; ISNTM, intrastromal natamycin; Fu, Fusarium; AS, Aspergillus; Ca, Candida; AI, Alternaria; Cu, Curvularia.

Table 2 Baseline Features in All Three Groups

\begin{tabular}{|c|c|c|c|c|c|c|}
\hline Drug & $\begin{array}{l}\text { Epithelial } \\
\text { Defect } \\
\text { Mean士SD }\end{array}$ & $\begin{array}{l}\text { Stromal } \\
\text { Infiltrate } \\
\text { Mean士SD }\end{array}$ & $\begin{array}{l}\text { Presence of } \\
\text { Hypopyon }\end{array}$ & $\begin{array}{l}\text { Height of } \\
\text { Hypopyon } \\
\text { Mean士SD }\end{array}$ & $\begin{array}{l}\text { Retro Corneal } \\
\text { Plaque }\end{array}$ & $\begin{array}{l}\text { Visual Acuity } \\
\text { (LogMAR) } \\
\text { Mean } \pm \text { SD }\end{array}$ \\
\hline ISVCZ $(n=20)$ & $3.0 \pm 1.1$ & $4.0 \pm 1.1$ & 14 & I. $15 \pm 0.49$ & $9 / 20$ & $2.2 \pm 0.3$ \\
\hline ISAMB $(n=20)$ & $3.5 \pm 1.15$ & $4.3 \pm 1.05$ & 13 & I. $13 \pm 0.50$ & $8 / 20$ & $2.3 \pm 0.4$ \\
\hline ISNTM $(n=20)$ & $3.0 \pm 1.02$ & $4.1 \pm 1.1$ & 13 & $1.30 \pm 0.73$ & $8 / 20$ & $2.2 \pm 0.4$ \\
\hline $\mathrm{P}$-value & 0.75 & 0.49 & 0.8 & 0.85 & 0.9 & 0.70 \\
\hline
\end{tabular}

Abbreviations: ISVCZ, intrastromal voriconazole; ISAMB, intrastromal amphotericin; ISNTM, intrastromal natamycin.

a significant difference between the three groups, $p=0.02$ and a hazard ratio of 1.44. (Figure 3). Mean time taken for complete resolution of stromal infiltrates and the mean scar size were comparable in all groups.

\section{Secondary Outcome}

The mean BCVA at baseline was similar in all three groups $(\mathrm{p}=0.70$ ). The mean BCVA at 6 months with perprotocol analysis was $1.1 \pm 0.1 \log \mathrm{MAR}$ units in the ISVCZ group, $1.1 \pm 0.1 \log$ MAR units in the ISAMB group and 1.0 $\pm 0.1 \log$ MAR units in the ISNTM group ( $\mathrm{p}=0.59)$, implying a comparable final visual outcome in all groups. Using intention to treat analysis, the mean BCVA at 6 months was $1.2 \pm 0.3 \log$ MAR units in the ISVCZ group, $1.3 \pm 0.4$ in the ISAMB group and $1.2 \pm 0.3$ in the ISNTM group, and was not statistically significant $(\mathrm{p}=0.54)$ (Tables 4 and 5 ).

\section{Complications and Sequelae}

None of the patients in our study had iatrogenic perforations, endophthalmitis, allergic reactions or glaucoma. No defects or damage to the epithelium, intrastromal deposits or worsening of conjunctival congestion were documented throughout the entire study. Deep vascularization was appreciated in $5 / 19$ eyes (26\%) in the ISVCZ group, 10/ 18 eyes (55\%) in the ISAMB group and 6/19 eyes (31\%) in the ISNTM group $(\mathrm{p}=0.02)$. Presence of cataract was noted in $14 / 19,15 / 18$ and $13 / 19$ eyes in the ISVCZ, ISAMB and ISNTM groups, respectively $(p=0.4)$ at 6-month follow-up (Table 6).

\section{Discussion}

The overall success rate of intrastromal antifungal agents in treating recalcitrant fungal keratitis in the present study was $93.33 \%$. All drugs demonstrated comparable efficacy $(\mathrm{p}=0.8$ ), with individual success rates of $95 \%, 90 \%$ and $95 \%$ with ISVCZ, ISAMB and ISNTM, respectively. The three agents were also comparable in final visual outcome, scar size and rate of healing. To the best of our knowledge, this is the first study comparing the efficacy and safety of three different antifungal agents employed for intrastromal injection.

The rate of healing with ISVCZ in our study (95\%) corresponded with that of multiple previous studies reporting success rates of 66.6 to $100 \%$ with the same agent in recalcitrant fungal keratitis. ${ }^{4,5,17,18,19}$ However, this was in contrast to a recent study by Narayana et al, who demonstrated no added advantage of ISVCZ in the management of moderate to severe fungal corneal ulcers. ${ }^{20}$ We believe these conflicting results could be explained by the ethnic variability and regional differences in microbiological profile in both studies. While Narayan et al most commonly isolated Fusarium sp., Aspergillus sp. was traced most 


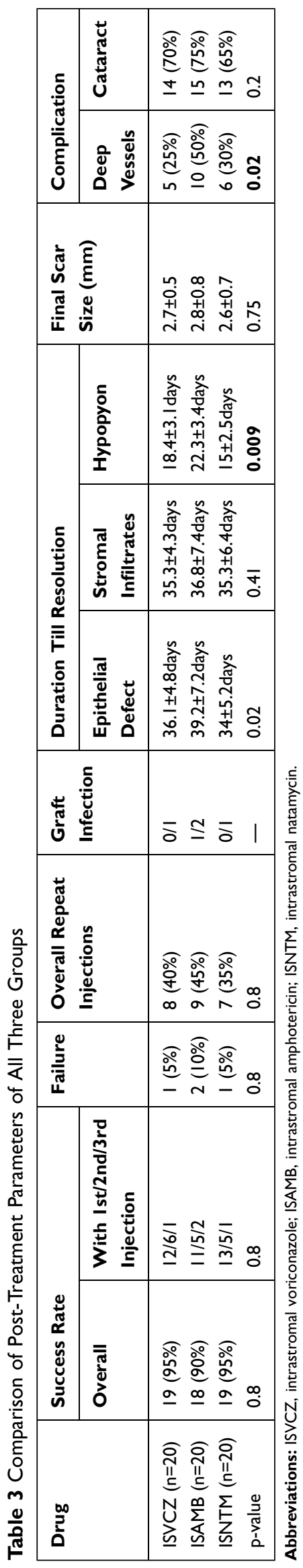

commonly in our study, a finding corresponding with prior studies undertaken in North India. ${ }^{21}$ We believe that assessing prior sensitivity of the organism to the treating drug may contribute to improving surgical results. Moreover, the conclusions in the study by Narayana et al are difficult to interpret as microbiological cure was considered the primary outcome measure in their study compared to clinical resolution in our study.

The healing rate of $90 \%$ with ISAMB in the present study is also comparable to that of Nada et al's study $(83 \%))^{7}$ However, the need for administering repeat injections was higher in this group. This could be due to delayed healing, as depicted by slow resolution of epithelial defect, stromal infiltrates and hypopyon, all of which could have ultimately resulted in exaggerated inflammation and therefore larger final scar size, and significantly higher propensity to develop deep vascularization with ISAMB. Although previous studies have reported toxicity of AMB to endothelial cells, ours is the first study reporting increased incidence of corneal vascularization with its intrastromal delivery. ${ }^{22}$ While deep vascularization makes the cornea a high risk bed for corneal grafting in the future, the actual effect of these agents on graft survival needs to be ascertained with larger and longer studies. Till further studies validate or refute our results, we recommend some caution before advocating ISAMB in eyes planned for keratoplasty in the future.

To date, only Mimouni et al have commented on the effect of ISNTM in recalcitrant fungal keratitis cases, and in an animal model. ${ }^{8}$ While their experimental study showed little beneficial effect of additive ISNTM 5\% over topical therapy alone in the treatment of Fusarium keratitis, we experienced a $95 \%$ cure rate with ISNTM. This could result from the varied composition of NTM utilized in our study. Mimouni et al directly injected topical NTM 5\% drops, while we specifically injected a unique cyclodextrin-combined-NTM formulated aseptically in our pharmacology department, which could have enhanced drug penetration at targeted sites, thereby improving treatment results. A similar phenomenon may have been experienced by O'Day 30 years ago, when identifying poor intracorneal and aqueous levels of NTM ( $5 \%$ microfine suspension) in rabbit eyes after its intrastromal injection. ${ }^{10}$ This unique composition may also be the reason that we did not encounter any precipitate formation with the drug, a complication commonly witnessed with the emulsion form of topical NTM. ${ }^{8}$ Not only the novel composition but also the variable in vivo 


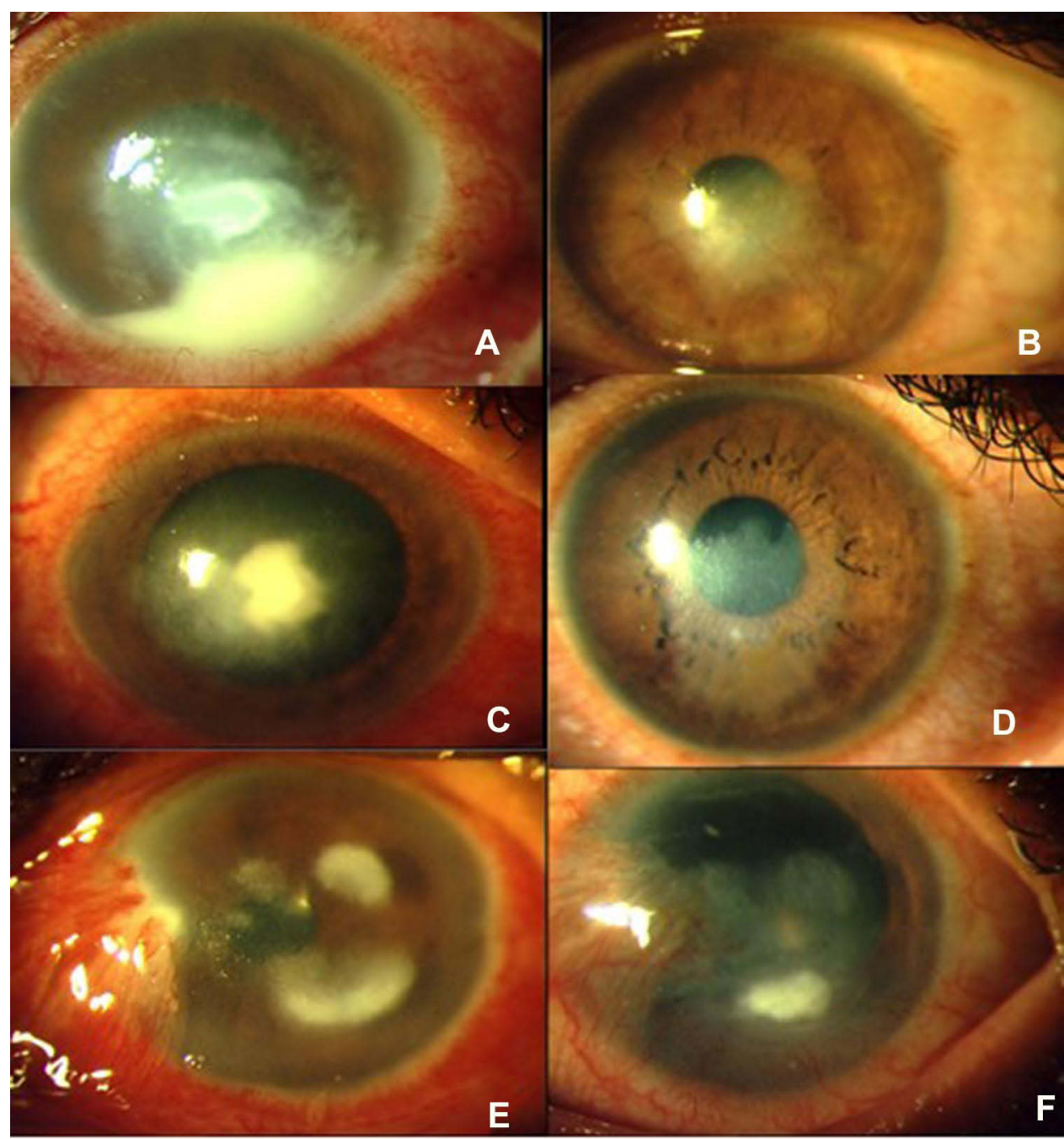

Figure 2 (A, C and E) Clinical photographs of recalcitrant fungal keratitis at day 0 in voriconazole, natamycin and amphotericin B groups, respectively. (B, D and F) Clinical photographs of cases (depicted in A, C and $\mathbf{E}$ ) after healing in the voriconazole, natamycin and amphotericin B groups, respectively.

responsiveness of Aspergillus and Fusarium to ISNTM in human eyes could have contributed to higher cure rates with ISAMB. Collectively, these factors could have contributed to the lower number of repeat injections and a significantly earlier resolution of epithelial defect as well as the hypopyon in the ISNTM group, all suggestive of lessened inflammation as indicated by a smaller scar size in the same group. However, further studies are required to validate these findings, as studies evaluating the utility of ISNTM in recalcitrant fungal keratitis in human eyes are presently minimal. Nevertheless, considering the paucity of antifungal agents, encouraging results with a novel composition of an already existing drug may open up a new arena for management of complex recalcitrant fungal keratitis cases.
A large number of studies have also reported on the potential use of intrastromal injections for fungal keratitis after penetrating keratoplasty and kerato-refractive procedures. ${ }^{23-25}$ However, as this study was the first of its kind, we limited the study to eyes with fungal keratitis not subjected to prior corneal surgeries. Moreover, it would not have produced reliable results due to a fewer number of patients. Based on the success of the intrastromal agents in the present study and a literature review, we believe that intrastromal injections might be helpful in post-PKP eyes also. However, further studies are needed in this area.

Intracameral injections of antifungal drugs along with intrastromal injections have been found to be effective in the early resolution of endothelial plaque, but in our study, 

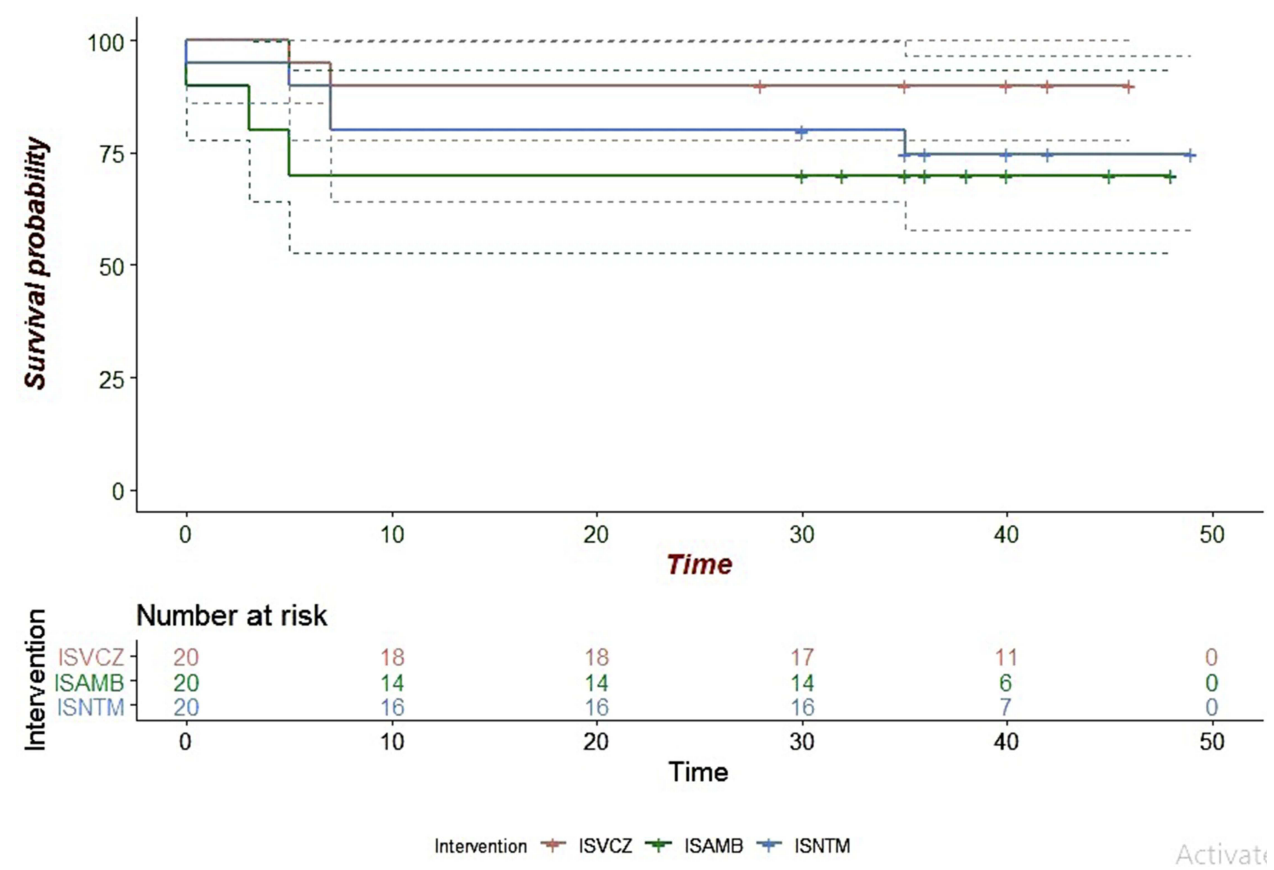

Figure 3 Kaplan-Meier analysis showing the duration of re-epithelialization.

Notes: The Kaplan-Meier curve shown has three intervention groups: ISVCZ, ISAMB and ISNTM. The number of people at risk at time point 0 is 20 in each intervention group. From the p-value (0.02) and hazard ratio (I.44) shown in the graph, it is clear that there is a significant difference between the three intervention groups, with a higher risk of failure in the ISAMB group.

as natamycin was being given for the first time, intracameral injections were avoided. Also, to maintain uniformity in the management of reliable results, only intrastromal injections were administered in all patients. ${ }^{26}$ Although in the previous study by Lalitha et al the presence of hypopyon was found to be a significant risk factor for treatment

Table 4 Post-Treatment (BCVA) Using ITT and Per-Protocol Analysis Method

\begin{tabular}{|l|l|l|l|l|l|}
\hline Analysis Method & BCVA (logMAR) & ISVCZ $^{\mathbf{a}}$ & ISAMB $^{\mathbf{b}}$ & ISNTM $^{\mathbf{c}}$ & $\mathbf{P}^{-v a l u e}$ \\
\hline ITT analysis & Baseline & $2.27 \pm 0.34$ & $2.24 \pm 0.40$ & $2.23 \pm 0.35$ & 0.9 \\
& Final follow-up & $1.2 \pm 0.38$ & $1.35 \pm 0.47$ & $1.2 \pm 0.37$ & 0.54 \\
\hline Per-protocal analysis & Baseline & $2.27 \pm 0.34$ & $2.24 \pm 0.40$ & $2.23 \pm 0.35$ & 0.9 \\
& Final follow-up & $1.1 \pm 0.1$ & $1.1 \pm 0.1$ & $1.0 \pm 0.1$ & 0.59 \\
\hline
\end{tabular}

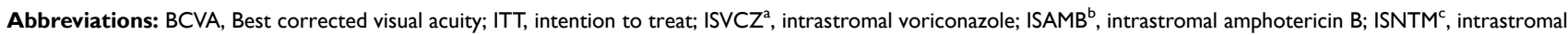
natamycin.

Table 5 Comparison of BCVA for Central, Paracentral and Peripheral Ulcers (logMAR)

\begin{tabular}{|l|l|l|l|l|}
\hline Ulcer Location & ISVCZ & ISAMB & ISNTM & P value \\
\hline Central & $\mathrm{n}=2$ & $\mathrm{n}=2$ & $\mathrm{n}=2$ & \\
Baseline & $2.7 \pm 0.22$ & $2.7 \pm 0.5$ & $2.5 \pm 0.12$ & 0.9 \\
Final follow-up & $2.15 \pm 0.12$ & $2.7 \pm 0.14$ & $2.08 \pm 0.13$ & 0.1 \\
Paracentral & $\mathrm{n}=13$ & $\mathrm{n}=14$ & $\mathrm{n}=14$ & \\
Baseline & $2.23 \pm 0.7$ & $2.3 \pm 0.3$ & $2.25 \pm 0.34$ & 0.9 \\
Final follow-up & $1.28 \pm 0.6$ & $1.3 \pm 0.5$ & $1.27 \pm 0.7$ & 0.7 \\
Peripheral & $\mathrm{n}=5$ & $\mathrm{n}=4$ & $\mathrm{n}=4$ & \\
Baseline & $1.74 \pm 0.6$ & $1.76 \pm 0.3$ & $1.85 \pm 0.4$ & 0.7 \\
Final follow-up & $\mathrm{I} \pm 0.2$ & $1.12 \pm 0.6$ & $0.8 \pm 0.3$ & 0.3 \\
\hline
\end{tabular}

Abbreviations: ISVCZ, intrastromal voriconazole; ISAMB, intrastromal amphotericin B; ISNTM, intrastromal natamycin; BCVA, best corrected visual acuity. failure, none of our patients who experienced treatment failure had hypopyon. ${ }^{27}$

Early deep lamellar keratoplasty has also been found to be effective in patients with fungal keratitis who are nonresponsive to medical therapy. ${ }^{28}$

The major limitations of this study include its relatively small sample size, the lack of a standard, clearly defined dose and pharmacokinetics of ISNTM, as well as its added cost. Lack of control groups (receiving only topical NTM 5\%) in our study could be attributed to our ethical limitation of allowing recalcitrant fungal keratitis to take its own fate from lack of added interventions. 
Table 6 Clinical Details of Patients with Treatment Failure

\begin{tabular}{|l|l|l|l|}
\hline Parameters & $\begin{array}{l}\text { ISVCZ, } \\
\mathbf{n = I}\end{array}$ & $\begin{array}{l}\text { ISAMB, } \\
\mathbf{n = 2}\end{array}$ & $\begin{array}{l}\text { ISNTM, } \\
\mathbf{n = I}\end{array}$ \\
\hline $\begin{array}{l}\text { Size of epithelial defect } \\
(\mathrm{mm})\end{array}$ & $6 * 6$ & $\begin{array}{l}5 * 5.5 \\
5 * 4\end{array}$ & $5.5 * 5$ \\
\hline Hypopyon height (mm) & $\mathrm{I}$ & $\begin{array}{l}0.5 \\
1.5\end{array}$ & $\mathbf{I}$ \\
\hline BCVA Log MAR & 2.7 & $\begin{array}{l}\text { Case I-2.7 } \\
\text { Case 2-2.7 }\end{array}$ & 2.3 \\
\hline $\begin{array}{l}\text { Duration of symptoms } \\
\text { (days) }\end{array}$ & 30 & $\begin{array}{l}\text { Case I-15 } \\
\text { Case 2-14 }\end{array}$ & 14 \\
\hline Time to failure (days) & 3 & $\begin{array}{l}\text { Case I-7 } \\
\text { Case 2-3 }\end{array}$ & 3 \\
\hline Depth of ulcer & $>75 \%$ & $\begin{array}{l}\text { Case I-75\% } \\
\text { Case2 } \\
>75 \%\end{array}$ & 3 \\
\hline
\end{tabular}

Abbreviations: ISVCZ, intrastromal voriconazole; ISAMB, intrastromal amphotericin B; ISNTM, intrastromal natamycin.

To conclude, intrastromal antifungal agents can be a safe and useful adjunct to standard therapy for management of recalcitrant fungal keratitis, specifically caused by the filamentary fungi. However, regional differences in microbiological profile have to be considered before prescribing them. Based on our study results, ISVCZ seems to be the best first-line anti-stromal agent. However, novel composition of NTM holds promising results and its applicability for treating recalcitrant fungal keratitis can be explored in the future. However, larger long-term, randomized comparative trlals are awaited to determine the most efficacious and safest intrastromal agent.

\section{Data Sharing Statement}

The authors do not intend to share the study data.

\section{Financial Disclosure(s)}

None of the authors have any proprietary or commercial interest in the materials discussed in this article.

\section{Disclosure}

T Velpandian reports a patent Novel Topical Natamycin Formulation for Ocular Antifungal Therapy (1950/DEL/ 2011) pending to the All India Institute of Medical Sciences, New Delhi. The authors report no other conflicts of interest in this work.

\section{References}

1. Krachmer JH, Mannis HJ, Holland EJ, Alfonso FC, Rosa RH. Fungal keratitis. In: Krachmer JH, Mannis HJ, Holland EJ, editors. Cornea and External Diseases: Clinical Diagnosis and Management. St Louis: CV Mosby; 1997:1253-1266.

2. Roozbahani M, Hammersmith KM, Nagra PK, et al. Therapeutic penetrating keratoplasty: a retrospective review. Eye Contact Lens. 2018;44:S433-S441. doi:10.1097/ICL.0000000000000522

3. Zhu Z, Zhang H, Yue J, et al. Antimicrobial efficacy of corneal cross-linking in vitro and in vivo for Fusarium solani: a potential new treatment for fungal keratitis. BMC Ophthalmol. 2018;18:65. doi:10.1186/s12886-018-0727-0

4. Chen Y, Gao M, Duncan JK, et al. Excisional keratectomy combined with focal cryotherapy and amniotic membrane inlay for recalcitrant filamentary fungal keratitis: a retrospective comparative clinical data analysis. Exp Ther Med. 2016;12:3014-3020. doi:10.3892/etm.2016.3699

5. Sharma N, Agarwal P, Sinha R, et al. Evaluation of intrastromal voriconazole injection in recalcitrant deep fungal keratitis: case series. $\quad B r \quad J$ Ophthalmol. 2011;95:1735-1737. doi:10.1136/ bjo.2010.192815

6. Prakash G, Sharma N, Goel M, et al. Evaluation of intrastromal injection of voriconazole as a therapeutic adjunctive for the management of deep recalcitrant fungal keratitis. Am J Ophthalmol. 2008;146:56-59. doi:10.1016/j.ajo.2008.02.023

7. Nada WM, Al Aswad MA, El-Haig WM. Combined intrastromal injection of amphotericin B and topical fluconazole in the treatment of resistant cases of keratomycosis: a retrospective study. Clin Ophthalmol Auckl NZ. 2017;11:871-874. doi:10.2147/OPTH. S135112

8. Mimouni M, Tam G, Paitan Y, et al. Safety and efficacy of intrastromal injection of 5\% natamycin in experimental fusarium keratitis. $J$ Ocul Pharmacol Ther off $J$ Assoc Ocul Pharmacol Ther. 2014;30:543-547. doi:10.1089/jop.2014.0004

9. Olusanya B, Onoja G, Ibraheem W, et al. Profile of patients presenting at a low vision clinic in a developing country. BMC Ophthalmol. 2012;12:31. doi:10.1186/1471-2415-12-31

10. O'Day DM, Head WS, Robinson RD, et al. Corneal penetration of topical amphotericin B and natamycin. Curr Eye Res. 1986;5:877-882. doi:10.3109/02713688609029240

11. Kaur IP, Rana C, Singh H. Development of effective ocular preparations of antifungal agents. $J$ Ocul Pharmacol Ther off $J$ Assoc Ocul Pharmacol Ther. 2008;24:481-493. doi:10.1089/jop.2008.0031

12. Foster CS, Lass JH, Moran-Wallace K, et al. Ocular toxicity of topical antifungal agents. Arch Ophthalmol Chic Ill 1960. 1981;99:1081-1084. doi:10.1001/archopht.1981.03930011081019

13. Isipradit S. Efficacy of fluconazole subconjunctival injection as adjunctive therapy for severe recalcitrant fungal corneal ulcer. J Med Assoc Thail Chotmaihet Thangphaet. 2008;91:309-315.

14. Kaushik S, Ram J, Brar GS, et al. Intracameral amphotericin B: initial experience in severe keratomycosis. Cornea. 2001;20:715-719. doi:10.1097/00003226-200110000-00009

15. Kuriakose T, Kothari M, Paul P, et al. Intracameral amphotericin $\mathrm{B}$ injection in the management of deep keratomycosis. Cornea. 2002;21:653-656. doi:10.1097/00003226-200210000-00004

16. Yoon K-C, Jeong I-Y, Im S-K, et al. Therapeutic effect of intracameral amphotericin $\mathrm{B}$ injection in the treatment of fungal keratitis. Cornea. 2007;26:814-818. doi:10.1097/ICO.0b013e31806c791e

17. Sharma N, Chacko J, Velpandian T, et al. Comparative evaluation of topical versus intrastromal voriconazole as an adjunct to natamycin in recalcitrant fungal keratitis. Ophthalmology. 2013;120:677-681. doi:10.1016/j.ophtha.2012.09.023

18. Siatiri H, Daneshgar F, Siatiri N, et al. The effects of intrastromal voriconazole injection and topical voriconazole in the treatment of recalcitrant Fusarium keratitis. Cornea. 2011;30:872-875. doi:10.1097/ICO.0b013e3182100993 
19. Kalaiselvi G, Narayana S, Krishnan T, et al. Intrastromal voriconazole for deep recalcitrant fungal keratitis: a case series. $\mathrm{Br}$ $J$ Ophthalmol. 2015;99:195-198. doi:10.1136/bjophthalmol-2014305412

20. Narayana S, Krishnan T, Ramakrishnan S, et al. Mycotic antimicrobial localized injection: a randomized clinical trial evaluating intrastromal injection of voriconazole. Ophthalmology. 2019;126 (8):1084-1089. doi:10.1016/j.ophtha.2019.03.020

21. Chowdhary A, Singh K. Spectrum of fungal keratitis in North India. Cornea. 2005;24:8-15. doi:10.1097/01.ico.0000126435.25751.20

22. Sharma B, Kataria P, Anand R, et al. Efficacy profile of intracameral amphotericin B. The often forgotten step. Asia Pac J Ophthalmol (Phila). 2015;4(6):360-366. doi:10.1097/APO.0000000000000107

23. Cavallini GM, Ducange P, Volante V, Benatti C. Successful treatment of Fusarium keratitis after photo refractive keratectomy. Indian J Ophthalmol. 2013;61(11):669-671. doi:10.4103/0301-4738.120213

24. Eleiwa T, Ozcan E, Abdelrahman S, et al. Case series of perforated keratomycosis after laser-assisted in situ keratomileusis. Case Rep Ophthalmol Med. 2020;2020:7237903.
25. Garcia-Valenzuela E, Song CD. Intracorneal injection of amphothericin B for recurrent fungal keratitis and endophthalmitis. Arch Ophthalmol. 2005;123(12):1721-1723. doi:10.1001/archopht.123. 12.1721

26. Kitazawa K, Fukuoka H, Inatomi T, Aziza Y, Kinoshita S, Sotozono C. Safety of retrocorneal plaque aspiration for managing fungal keratitis. Jpn J Ophthalmol. 2020;64(2):228-233. doi:10.1007/ s10384-020-00718-3

27. Lalitha P, Prajna NV, Kabra A, Mahadevan K, Srinivasan M. Risk factors for treatment outcome in fungal keratitis. Ophthalmology. 2006;113(4):526-530. doi:10.1016/j.ophtha.2005.10.063

28. Sabatino F, Sarnicola E, Sarnicola C, Tosi GM, Perri P, Sarnicola V; Medscape. Early deep anterior lamellar keratoplasty for fungal keratitis poorly responsive to medical treatment. Eye (Lond). 2017;31 (12):1639-1646. doi:10.1038/eye.2017.228
Clinical Ophthalmology

\section{Publish your work in this journal}

Clinical Ophthalmology is an international, peer-reviewed journal covering all subspecialties within ophthalmology. Key topics include: Optometry; Visual science; Pharmacology and drug therapy in eye diseases; Basic Sciences; Primary and Secondary eye care; Patient Safety and Quality of Care Improvements. This journal is indexed on PubMed

\section{Dovepress}

Central and CAS, and is the official journal of The Society of Clinical Ophthalmology (SCO). The manuscript management system is completely online and includes a very quick and fair peer-review system, which is all easy to use. Visit http://www.dovepress.com/ testimonials.php to read real quotes from published authors. 